\title{
The Weighting Algorithms for the Landweber Method in Electromagnetic Tomography
}

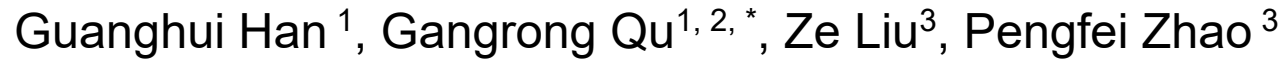 \\ ${ }^{1}$ School of Science, Beijing Jiaotong University, Beijing 100044, China \\ 2 Beijing Center for Mathematics and Information Interdisciplinary Sciences (BCMIIS) Beijing \\ 100048, China \\ ${ }^{3}$ School of Electronics and Information Engineering, Beijing Jiaotong University, Beijing 100044, \\ China \\ *Imingliangl@163.com
}

\begin{abstract}
Due to ill-posed of the image reconstruction in Electromagnetic tomography (EMT), the condition number of the sensitivity matrix is high and the reconstructed results are sensitive to the measurement errors. In this paper, two weighting matrices are proposed to improve the condition number and two modified Landweber methods are derived. Numerical simulations demonstrate the feasibility of the modified methods.
\end{abstract}

Keywords: Electromagnetic tomography, Image reconstruction, Sensitivity matrix, Landweber iteration, Gain factor.

\section{Introduction}

Electromagnetic tomography (EMT) is a non-destructive imaging technology which is to reproduce the distribution of conductivity or permeability in an object by the measurement data from detection coils. Based on the sensitivity theory [1,2], the forward problem of the EMT system is modeled as the following linear systems

$$
S g=z,
$$

where $g=\left(g_{1}, g_{2}, \cdots, g_{N}\right)^{T}$ denotes the conductivity distribution, $z=\left(z_{1}, z_{2}, \cdots, z_{M}\right)^{T}$ is the measurement data, $S=\left(s_{i j}\right)_{M \times N}$ is the sensitivity matrix. Therefore, the image reconstruction is obtained by solving the linear systems. In practical problems, iterative methods are usually used to solve the Eq. (1). In the image reconstruction of EMT system, many iterative algorithms have been developed because of its effectiveness and convenience [3]. Iterative algorithms mainly include Newton Raphson iteration, Landweber iteration, Tikhonov iterative, etc. [3-5].

In this paper, we analyze the condition number of the sensitivity matrix and we find two weighting matrices to reduce the condition number. Based on this, this paper gives the weighting Landweber scheme which can improve the quality of the reconstructed images. The organization of this paper is as follows. In section 2, two weighting matrices are proposed to reduce the condition number. Section 3 gives two modified algorithms based on these two weighting matrices. In section 4, numerical simulations are performed to demonstrate the validity of the modified algorithms. Finally, we discuss some relevant conclusions.

\section{Reducing Condition Number}

In general, the sensitivity matrix $S$ is neither symmetric nor positive definite. Hence, we first regularize the Eq. (1), which is multiplied by matrix $S^{T}$,

$$
S^{T} S g=S^{T} z
$$


where ${ }^{T}$ refers to the transpose of the matrix $S$. The Eq. (2) is always solvable. The different positive eigenvalues of $S^{T} S$ are denoted by $\left\{\lambda_{k}\right\}_{k=1}^{m}$, and the order of magnitude is $\lambda_{1}>\lambda_{2}>\cdots>\lambda_{m}>0$. The multiplicity of $\lambda_{k}$ is marked as $p_{k}(1 \leq k \leq m)$.

Definition 2.1 The condition number of the matrix $S^{T} S$ is:

$$
\kappa\left(S^{T} S\right)=\frac{\lambda_{1}}{\lambda_{m}}
$$

where $\lambda_{m}, \lambda_{1}$ are the smallest and largest positive eigenvalues of matrix $S^{T} S$, respectively.

By the property of monotonic decreasing of eigenvalues, $\lambda_{1}>\cdots>\lambda_{m}>0$, we want to find a monotonic increasing sequence. Then let the corresponding item of the two sequences be multiply, so that the distance between the largest eigenvalue and the smallest eigenvalue is reduced. This monotonic increasing sequence is assumed to be $0<\left(\omega-\lambda_{1}\right)<\cdots<\left(\omega-\lambda_{m}\right),\left(\omega>\lambda_{1}\right)$. Given each item of this sequence is treated as an eigenvalue, the corresponding matrix is $\left(\omega I-S^{T} S\right),\left(\omega>\lambda_{1}\right)$, where $I$ is $N \times N$ identity matrix. The matrix $\left(\omega I-S^{T} S\right)$ is symmetric positive definite. If the largest eigenvalue $\lambda_{1}$ is only computed, then we let $\omega=2 \lambda_{1}$. If the largest eigenvalue $\lambda_{1}$ and the smallest positive eigenvalue $\lambda_{m}$ are all obtained, then the parameter is taken as $\omega=\lambda_{1}+\lambda_{m}$. Thus, using the symmetric positive definite matrices $\left(2 \lambda_{1} I-S^{T} S\right)$ and $\left(\left(\lambda_{1}+\lambda_{m}\right) I-S^{T} S\right)$ to multiply the Eq. (2), we obtain

$$
\begin{aligned}
& \left(2 \lambda_{1} I-S^{T} S\right) S^{T} S g=\left(2 \lambda_{1} I-S^{T} S\right) S^{T} z, \\
& \left(\left(\lambda_{1}+\lambda_{m}\right) I-S^{T} S\right) S^{T} S g=\left(\left(\lambda_{1}+\lambda_{m}\right) I-S^{T} S\right) S^{T} z .
\end{aligned}
$$

Since the matrices $\left(2 \lambda_{1} I-S^{T} S\right)$ and $\left(\left(\lambda_{1}+\lambda_{m}\right) I-S^{T} S\right)$ are nonsingular, the Eqs. (4) and (5) have the same solutions as the Eq. (2).

\section{Modifying the Landweber Method}

The principle of iteration is proposed in [4]. Yang W.Q. et al. regard $A_{0}$ as an approximation to $S^{-1}$ and give an iterative formula

$$
g_{k+1}=g_{k}+A_{0}\left(z-S g_{k}\right) .
$$

At first, the Landweber method was used to solve the Fredholm integral equation [2]. In the formula (6), $A_{0}$ is replaced by $\alpha S^{T}$. Then the Landweber method is obtained

$$
g_{k+1}=g_{k}+\alpha S^{T}\left(z-S g_{k}\right),
$$

where the parameter $\alpha$ is called as the gain factor. A sufficient condition for convergence is given by $\left\|\alpha S^{T} S\right\|<2$, from which the common value of the gain parameter is set to be $0<\alpha<2 / \lambda_{1}$ [11]. However, the optimal value of the gain factor is $\alpha_{o p t}=2 /\left(\lambda_{1}+\lambda_{m}\right)$ for the Landweber method (7), where $\lambda_{m}$ and $\lambda_{1}$ are the smallest and largest positive eigenvalues of $S^{T} S$ [6]. 
Based on the above analysis of reducing the condition number, we propose that $A_{0}$ is given by $\alpha\left(2 \lambda_{1} I-S^{T} S\right) S^{T}$ and $\alpha\left[\left(\left(\lambda_{1}+\lambda_{m}\right) I-S^{T} S\right) S^{T} S\right] S^{T}$, respectively. Hence, two modified Landweber iterations can be obtained

$$
\begin{gathered}
g_{k+1}=g_{k}+\alpha\left(2 \lambda_{1} I-S^{T} S\right) S^{T}\left(z-S g_{k}\right), \\
g_{k+1}=g_{k}+\alpha\left[\left(\lambda_{1}+\lambda_{m}\right) I-S^{T} S\right] S^{T}\left(z-S g_{k}\right) .
\end{gathered}
$$

\section{Numerical Simulations}

The simulation model is a typical single coil excitation mode of EMT, and 8 coils are arranged on the outer circumference of the pipe. The finite-element models are shown in Figure 1, in which there are three different types of the fields. The triangular element is used to divide the detected field. We use the Landweber method, Modified-1 and Modifi-ed-2 methods to reconstruct the images. The number of iterations is 200. In general, the image error (IE) is calculated for quantitative comparison of different iterative methods

$$
I E=\frac{\|g-\hat{g}\|}{\|g\|},
$$

where $\hat{g}$ and $g$ are the estimated and true conductivity or permeability vectors, respectively [5]. The lower the IE values, the closer the estimated conductivity values to the true ones. Firstly, the condition number of the coefficient matrix is $5.9872 \times 10^{3}$ for the Eq. (2). For the modified methods (4) and (5), the condition numbers are reduced to $2.9938 \times 10^{3}$ and $1.3968 \times 10^{3}$, respectively. The reduction ratio is $50 \%$ and $76.67 \%$. Secondly, from Figure 2, we see that the values of IE from the Modified-1 and Modified-2 methods are smaller than those from the Landweber method in all three different fields.
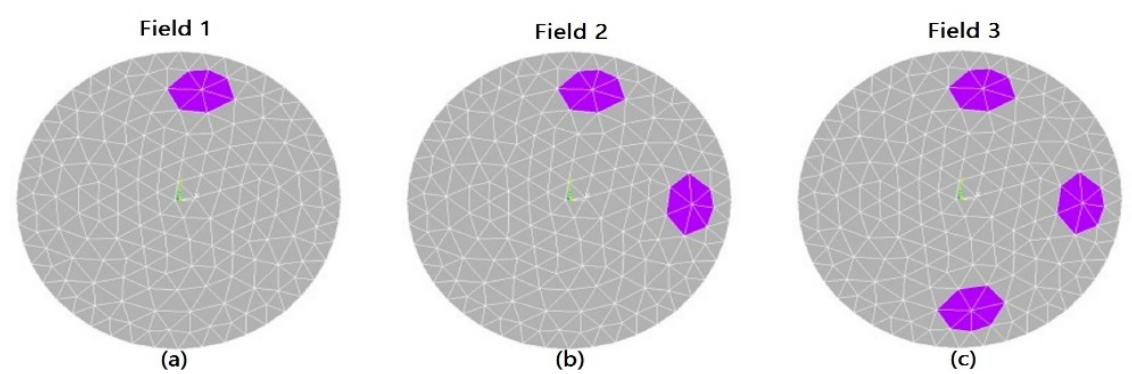

Figure 1. The finite-element models: (a) one target, (b) two targets, (c) three targets 

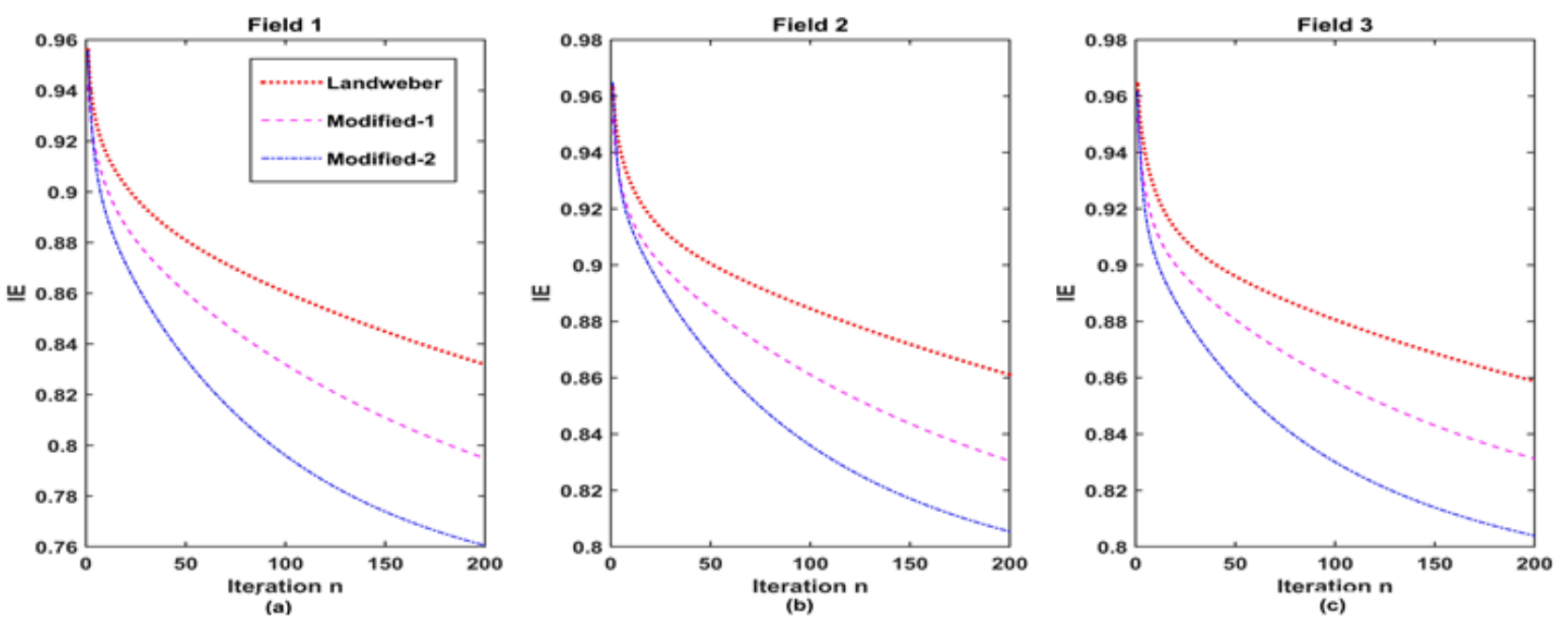

Figure 2. Image error histories generated from three iterative algorithms.

\section{Conclusion}

In this paper, the condition number of the regularized sensitivity matrix is reduced.

by weighting matrix. According to the method of weighting matrix, we have proposed two modified algorithms which can improve the equality of the reconstructed images. Numerical simulations have verified the feasibility of modified methods.

\section{Acknowledgments}

The authors are partially supported by the National Natural Science Foundations of China (61671004, 61271012, 61227002).

\section{References}

[1]. Geselowitz D.B., An application of electrocardiographic lead theory to impedance plethysmography, IEEE Transaction on Biomedical Engineering, (1971) BME-18(1), p. 38 -- 41.

[2]. Landweber L., An iteration formula for Fredholm integral equations of the first kind with application to the axially symmetric potential flow about elongate bodies of revolution, American Journal of Mathematics, (1951) 3 (73), p. 615 -- 624.

[3]. Liu S., Fu L., Yang W. Q., Optimization of an iterative image reconstruction algorithm for electrical capacitance tomography, Measurement Science and Technology, (1999) 10, p. L37-L39.

[4]. Yang W. Q., Sprink D. M., York T.A., McCann H., An image-reconstruction algorithm based on Landweber's iteration method for electrical-capacitance tomography, Measurement Science and Technology, (1999) 10, p. 1065--1069.

[5]. Pan T.S., Yagle A.E., Acceleration of Landweber-type algorithms by suppression of projection on the maximum singular vector, IEEE Trans. Med. Imaging, (1992) 11(4), p. 479--487.

[6]. Guanghui Han, Gangrong Qu, Ming Jiang, Relaxation Strategy for the Landweber Method, Signal Processing, (2016) 125, p. 87--96. 